\title{
Integración de TIC en la Formación Inicial Docente de la Universidad de Los Lagos
}

\author{
Brenda Angélica Lara Subiabre ${ }^{1}$, Yorka Tatiana Ortiz Ruiz ${ }^{2}$, Pedro Marcelo \\ Soto Alarcón ${ }^{3}$, Francisco Javier Núñez Romero ${ }^{4}$, Marisa Alejandra Lara \\ Escobar ${ }^{5}$, Francisco José Kroff Trujillo ${ }^{6}$
}

Resumen

El trabajo tuvo como propósito develar el estado en torno a la integración de TIC en la formación de estudiantes de las carreras de pedagogía de la Universidad de Los Lagos en los campus de Osorno y Puerto Montt de Chile. Para la recopilación de la información se utilizó la técnica de investigación documental, a través de la cual se analizan las mallas curriculares y los programas de estudio que son implementados en la formación de TIC en educación. Los resultados nos revelan que existen situaciones muy dispares en la formación en esta área, la que se expresa en diferencias significativas en la cantidad de horas designadas entre las carreras de ambos Campus. Además, según los estándares TIC para docentes en Chile, los estudiantes tendrían un alto porcentaje de horas de trabajo en la dimensión pedagógica, no así en la dimensión de gestión. Después del análisis realizado se propone una serie de medidas, entre las que se considera incorporar el trabajo con TIC al interior de las didácticas de la especialidad y en los procesos de prácticas, además de desarrollar instrumentos para medir el manejo de TIC tanto al ingreso como al finalizar el proceso formativo.

Palabras clave: TIC, Formación Inicial docente, estándares TIC docentes, educación superior

\begin{abstract}
The purpose of this work was to reveal the state around the integration of ICT in the training of students in the teaching majors at University of Los Lagos on the Osorno and Puerto Mont Campuses in Chile. For the compilation of the information we used the documentary research technique, though which the curricula implemented in the training of the ICT in Education. The results reveal that there are different training situations in this area, which is expressed in significant differences in the number of designated hours in the majors in both campuses. Besides, according to the ICT standards for teachers in Chile, students would have a high percentage of working hours in the pedagogical dimension not in the management dimension. After the analysis, we propose a series of alternatives, among which are considered incorporating the ICTs in the Didactics of the specialization and the practicum processes, and developing instruments to measure the use of ICT not only at the beginning but also at the end of the training process.
\end{abstract}

Key words: ICT, initial teacher training, ICT teachers' standards, higher education

\footnotetext{
1 Magíster en Ciencias de la Educación, Académica de la Universidad de Los Lagos, blara@ulagos.cl;

2 Magíster en Informática Educativa, académica Línea Informática Educativa. Escuela de Pedagogía, Universidad de Los Lagos, yortiz@ulagos.cl;

Técnico en informática, Docente área tecnologías Escuela de Pedagogía, Campus Chiquihue Universidad de Los Lagos, psoto@ulagos.cl;

4 Magíster en Pedagogía en Matemática y Computación, Profesor, Universidad de Los Lagos, fnunez@ulagos.cl;

Máster es TIC y Educación, Profesora, Universidad de Los Lagos, mlara@ulagos.cl;

Máster en Tecnologías para la Educación y el Conocimiento, francisco.kroff@ulagos.cl
} 


\section{Introducción}

Las Tecnologías de la Información y la Comunicación forman parte de la vida cotidiana de las personas y esta realidad requiere que las comunidades formen a las futuras generaciones para estar alfabetizadas tecnológicamente, utilizando las TIC para mejorar su calidad de vida. Ello solo será posible en la medida que los docentes asuman que es indispensable que incorporen las TIC a sus prácticas pedagógicas, y desarrollen en los escolares las habilidades para usarlas eficientemente. Para lo anterior se requiere que los estudiantes de carreras de pedagogía egresen de las instituciones de educación superior teniendo dominio de las TIC y habiendo desarrollado competencias que les permitan incorporarlas a su trabajo docente. Para ello es necesario que las instituciones de educación superior se examinen internamente y generen las condiciones más apropiadas para responder de forma eficiente a este desafío.

El estudio es la base para iniciar procesos de armonización curricular y sintonización de la formación de TIC en Educación en todas las carreras de pedagogía de la Universidad de Los Lagos.

La pregunta que orientó nuestro estudio fue: ¿cuál es el estado de integración de TIC en Educación en la formación de los estudiantes de las carreras de pedagogía en los campus Osorno y Puerto Montt de la Universidad de Los Lagos?
El objetivo general del estudio fue:

- Develar el estado de integración de TIC en educación en la formación de estudiantes de las carreras de pedagogía de la Universidad de Los Lagos en los campus de Osorno y Puerto Montt.

Los objetivos específicos fueron:

- Identificar la cantidad de asignaturas y horas que tributan en cada carrera a la formación de TIC en Educción.

- Identificar el grado de incorporación de las Competencias TIC en los programas de estudio de las asignaturas que tributan a la línea TIC en educación.

- Relacionar lineamientos ministeriales y referentes internacionales, para reconocer su grado de actualización y pertinencia en cuanto a contenidos de asignaturas que tributan a la línea de TIC en educación.

\subsection{Contexto}

Según el informe Mckinsey (2010) uno de los factores más relevantes de atender, para avanzar en mejorar la educación, es potenciar la formación inicial docente. Por su parte la UNESCO, ya en el año 1998, establecía en la declaración Mundial sobre Educación Superior en el siglo XXI que es indispensable mejorar los procesos de planificación, de didáctica y apropiación de TIC, en la formación

Volumen No. 1 Integración de TIC en la Formación Inicial Docente de la Universidad de Los Lagos 
profesional. Esto se ratificó en el año 2009, en la $2^{\circ}$ Conferencia Mundial, en donde se hizo un llamado a los Estados miembros para: "Apoyar la mayor integración de las tecnologías de la información y la comunicación para promover y enfrentar las crecientes demandas en la Educación Superior”.
Según un estudio realizado por la CEPAL (Brun, 2011) en el que participaron 16 países entre Latinoamericanos y del Caribe, los respectivos Ministerios de Educación identificaron los principales desafíos o dificultades actuales y futuras respecto a la inclusión de TIC en FID, a saber:

GRÁFICO 5

AMÉRICA LATINA Y EL CARIBE (16 PAISES) PRINCIPALES DESAFIOS ACTUALES Y FUTUROS PARA LA INTEGRACIÓN DE TIC EN LA FID SEGÚN LA VISIÓN DE LOS ACTORES (En porcentajes)

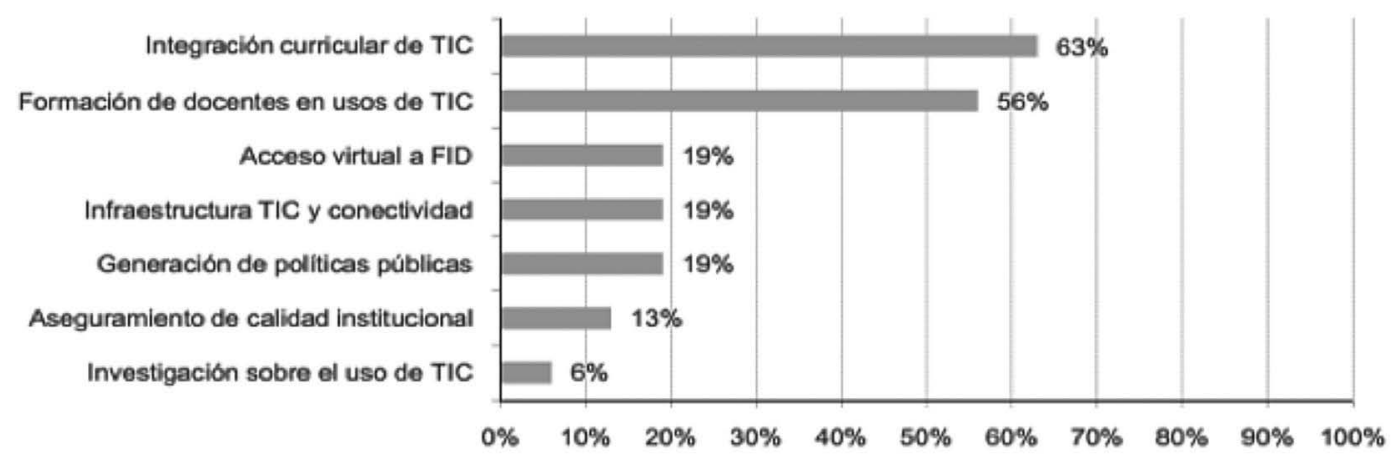

Fuente: Brun, 2011.

Así también, se identificaron áreas deficientes a través de fuentes secundarias, que indicaban lo siguiente:

- Información insuficiente o de difícil acceso

- Infraestructura y/o conectividad deficiente

- Capacitación docente escasa o incompleta

- Políticas públicas que abordan parcialmente la complejidad de la temática o que no garantizan su sostenibilidad
- Bajo nivel de coordinación y cooperación entre instituciones de FID.

- Débiles procesos de seguimiento y evaluación, y falta de indicadores aplicables al sector

- Escasa promoción de actividades de investigación.

Por otra parte un estudio realizado por Hepp (2012), reconoce que las barreras para el uso de las TIC en instituciones de formación inicial docente y en las escuelas, son las siguientes: 
[ 14 ] Universidad Católica de El Salvador.

- Infraestructura TIC de instituciones y cultura digital de sus docentes y estudiantes

- Los docentes, su actitud ante las TIC

- Las TIC en las disciplinas

- Vinculación con el Sistema Escolar

Sumado a los hallazgos anteriores, Silva y Astudillos (2012) detectan que las oportunidades para la inserción de las TIC en la Formación inicial docente, obedecen principalmente a:

- Desarrollar investigación para el diseño de modelos de observación/ evaluación de las aplicaciones y los aportes a la FID del uso de tecnología

- Contar con asesoría para proyectos de desarrollo e instalación de tecnología para innovar en las estrategias (metodológicas, didácticas, etc.) en nuevos modelos de formación docente

- Las tecnologías deberían integrarse transversalmente al currículo del programa

- Actualizar las mallas de planes de estudio para incorporar las tecnologías

En Chile, en las últimas décadas se han desarrollado e impulsado iniciativas de política pública, con la finalidad de reforzar la formación de los estudiantes de carreras de peda- gogía. El año 2006 el Centro de Educación y Tecnología del Ministerio de Educación (ENLACES) en colaboración con OREALC UNESCO, elaboraron la primera versión de los "Estándares TIC para la Formación Inicial Docente", los que fueron actualizados posteriormente. A partir del año 2010 se comienza a implementar el "Programa INICIA", que contempla la aplicación de un instrumento estandarizado a estudiantes del último año de carreras de pedagogía, con el fin de evaluar "Habilidades básicas TIC en ambiente pedagógico", Esta sección de la prueba se encuentra actualmente sin aplicar debido a su actualización. De acuerdo a los resultados obtenidos en esa prueba por los egresados de la Universidad de Los Lagos el año 2010, se aprecia que el desempeño del grupo evaluado fue de un 59\%, cinco puntos más atrás que el promedio nacional alcanzado (64\%).

\subsection{Competencias TIC para la Formación Inicial Docente}

El Ministerio de Educación en Chile (2008), ha definido las competencias TIC que deberían presentar los egresados de carreras de pedagogía, las que son divididas en cinco dimensiones:

a. Dimensión Pedagógica: demostrar formas de aplicar las TIC en el currículum escolar vigente como una forma de apoyar y expandir el aprendizaje y la enseñanza. 
b. Dimensión Técnica: dominio de las competencias asociadas al conocimiento general de las TIC y el manejo de las herramientas de productividad (procesador de texto, hoja de cálculo, presentador) e Internet, desarrollando habilidades y destrezas para el aprendizaje permanente de nuevos hardware y software.

c. Dimensión Gestión: uso de las TIC para apoyar su trabajo en el área administrativa, tanto a nivel de su gestión docente como de apoyo a la gestión del establecimiento.

\section{d. Dimensión Ética, Social y Legal: co-} nocen, se apropian y difunden entre sus estudiantes los aspectos éticos, legales y sociales relacionados con el uso de los recursos informáticos y contenidos disponibles en Internet; actuando de manera consciente y responsable respecto de los derechos, cuidados y respetos que deben considerarse en el uso de las TIC.

\section{e. Dimensión Desarrollo y Responsa-} bilidad Profesional: hacen uso de las TIC como medio de especialización y desarrollo profesional; informándose y accediendo a diversas fuentes para mejorar sus prácticas y facilitando el intercambio de experiencias que contribuyan mediante un proceso de reflexión con diversos actores educativos, para conseguir mejores procesos de enseñanza y aprendizaje.

En cada dimensión se desprenden las competencias y los respectivos criterios que indican cómo se manifiestan. A continuación se expone como se despliegan:

\section{DIMENSIÓN PEDAGÓGICA}

\begin{tabular}{|l|l|}
\hline Competencias & Criterios \\
\hline 1.1 Integrar TIC en la pla- & $\begin{array}{l}\text { 1.1.1 Planifica ambientes y experiencias de aprendizaje utilizando re- } \\
\text { sultados de estudios, buenas prácticas o estrategias probadas respecto } \\
\text { del uso de TIC. } \\
\text { nificación de ambientes y }\end{array}$ \\
$\begin{array}{l}\text { experiencias de aprendiza- } \\
\text { de actividades de aprendizaje y de acuerdo a los recursos disponibles. } \\
\text { je de los sectores curricu- } \\
\text { lares para agregar valor al } \\
\text { aprendizaje y al desarrollo } \\
\text { integral de los estudiantes. }\end{array}$ & $\begin{array}{l}\text { dizaje de los estudiantes de acuerdo a las oportunidades, normativas, } \\
\text { materiales y humanas del contexto de desempeño. }\end{array}$ \\
& 1.1.4 Diseña estrategias de evaluación utilizando recursos digitales \\
pertinentes a los aprendizajes esperados.
\end{tabular}




\begin{tabular}{|c|c|}
\hline \multicolumn{2}{|c|}{ 1. DIMENSIÓN PEDAGÓGICA } \\
\hline Competencias & Criterios \\
\hline \multirow{5}{*}{$\begin{array}{l}\text { 1.2 Integrar TIC en } \\
\text { la implementación de } \\
\text { ambientes y experiencias } \\
\text { de aprendizaje de los sec- } \\
\text { tores curriculares para } \\
\text { agregar valor al aprendizaje } \\
\text { y al desarrollo integral de } \\
\text { los estudiantes. }\end{array}$} & $\begin{array}{l}\text { 1.2.1 Implementa TIC en los ambientes y las experiencias de aprendiza- } \\
\text { je, acorde al contexto y los recursos tecnológicos disponibles. }\end{array}$ \\
\hline & $\begin{array}{l}\text { 1.2.2 Propicia en los estudiantes el desarrollo del pensamiento crítico y } \\
\text { otras funciones cognitivas de orden superior mediante la integración de } \\
\text { las TIC en el desarrollo de actividades de aprendizaje. }\end{array}$ \\
\hline & $\begin{array}{l}\text { 1.2.3 Desarrolla un ambiente de trabajo motivador y fomenta una dis- } \\
\text { posición positiva hacia la incorporación y uso de las TIC en el proceso } \\
\text { educativo. }\end{array}$ \\
\hline & $\begin{array}{l}\text { 1.2.4 Usa TIC para evaluar, de acuerdo a su pertinencia, los aprendizajes } \\
\text { de los estudiantes en los sectores }\end{array}$ \\
\hline & $\begin{array}{l}\text { 1.2.5 Usa TIC para retroalimentar los resultados de la evaluación para } \\
\text { que los estudiantes ajusten, propongan y acuerden mejoras para sus pro- } \\
\text { pios procesos de aprendizaje. }\end{array}$ \\
\hline \multirow{3}{*}{$\begin{array}{l}\text { 1.3 Incorporar sistemas } \\
\text { de información en línea y } \\
\text { de comunicación mediada } \\
\text { por computadores en la } \\
\text { implementación de expe- } \\
\text { riencias de aprendizaje con } \\
\text { los estudiantes. }\end{array}$} & $\begin{array}{l}\text { 1.3.1 Desarrolla experiencias para facilitar el aprendizaje instrumental } \\
\text { de sistemas electrónicos de información y de comunicación mediada } \\
\text { por computadores, pertinentes a las características de los estudiantes y } \\
\text { del contexto. }\end{array}$ \\
\hline & $\begin{array}{l}\text { 1.3.2 Desarrolla experiencias para el aprendizaje de estrategias de bús- } \\
\text { queda, localización, selección y almacenamiento de recursos de infor- } \\
\text { mación disponibles en sistemas electrónicos. }\end{array}$ \\
\hline & $\begin{array}{l}\text { 1.3.3 Desarrolla experiencias de aprendizaje que facilitan en los estu- } \\
\text { diantes la comprensión y reflexión de los alcances de la interacción en } \\
\text { modalidades de comunicación mediadas por computadores. }\end{array}$ \\
\hline
\end{tabular}

Volumen No. 1 Integración de TIC en la Formación Inicial Docente de la Universidad de Los Lagos 


\section{DIMENSIÓN TÉCNICA}

\section{Competencias}

2.1 Usar instrumentalmen-

te recursos tecnológicos, digitales y espacios virtuales en los procesos de enseñanza y aprendizaje.

2.2 Operar sistemas digitales de comunicación y de información, pertinentes y relevantes para los procesos de enseñanza y aprendizaje.

\section{Criterios}

2.1.1 Usa recursos tecnológicos y digitales para los procesos de enseñanza aprendizaje y para otras tareas docentes.

2.1.2 Usa recursos digitales de apoyo a los procesos de enseñanza aprendizaje y de gestión curricular en la planificación y realización de clases.

2.1.3 Construye espacios virtuales de colaboración usándolos para fines pedagógicos.

2.2.1 Formula e implementa estrategias de búsqueda, localización y selección de recursos de información a través de sistemas en línea.

2.2.2 Usa sistemas digitales de comunicación para interactuar con sus estudiantes y otros actores del sistema educacional, siguiendo protocolos propios de esta modalidad.

\section{DIMENSIÓN GESTIÓN}

\begin{tabular}{|c|c|}
\hline as & iterios \\
\hline $\begin{array}{l}\text { 3.1. Usar TIC para mejo- } \\
\text { rar y renovar procesos de } \\
\text { gestión curricular. }\end{array}$ & $\begin{array}{l}\text { 3.1.1 Integra el uso de TIC para potenciar el análisis e interpretación de } \\
\text { variables del desempeño académico y de eficiencia interna, y realizar ac- } \\
\text { ciones de mejoramiento. } \\
\text { 3.1.2 Usa TIC para programar, administrar y controlar el tiempo dedi- } \\
\text { cado a la labor docente. } \\
\text { 3.1.3 Colabora en la gestión de la organización, mantención y actual- } \\
\text { ización de los recursos digitales necesarios para la labor docente dentro } \\
\text { de la institución. } \\
\text { 3.1.4 Implementa, mediante el uso de entornos virtuales, estrategias de } \\
\text { comunicación y seguimiento del aprendizaje de los estudiantes que faci- } \\
\text { liten la interacción y el seguimiento de las actividades relacionadas con } \\
\text { materias educativas. } \\
\text { 3.1.5 Evalúa la pertinencia del uso de TIC para el logro de una gestión } \\
\text { curricular adecuada y oportuna, buscando periódicamente su actual- } \\
\text { ización y nuevas oportunidades para aplicarlas (nuevos ámbitos o con- } \\
\text { textos). }\end{array}$ \\
\hline
\end{tabular}

Brenda Angélica Lara Subiabre / Yorka Tatiana Ortiz Ruiz / Pedro Marcelo Soto Alarcón / Francisco Javier Núñez Romero | Volumen No. 1 Marisa Alejandra Lara Escobar / Francisco José Kroff Trujillo 
3.2 Usar TIC para mejorar y renovar la gestión institucional, en la relación con la comunidad y especialmente en la relación escuela-familia.
3.2.1 Usa TIC para participar en la gestión institucional, en acciones tales como la planificación e implementación de proyectos, estudios y acciones institucionales colectivas y de cooperación con el proyecto educativo institucional (PEI), que involucren a la comunidad y especialmente a las familias.

3.2.2 Usa los espacios virtuales existentes en el establecimiento para facilitar y promover la interacción con los padres, madres y apoderados.

3.2.3 Usa TIC para planificar e implementar actividades con los padres, madres y apoderados, para el acompañamiento académico de los estudiantes y para recoger información (formularios on line, encuestas, etc.)

\section{DIMENSIÓN SOCIAL, ETICA Y LEGAL}

\section{Competencias}

4.1 Integrar TIC para promover el desarrollo de habilidades sociales, nuevas formas de socialización y el desarrollo de ciudadanía digital.

\section{Criterios}

4.1.1 Promueve, a través de la implementación de ambientes de aprendizaje con TIC, el desarrollo de habilidades sociales para la participación y el aprendizaje colaborativo y en red.

4.1.2 Explora con los estudiantes las nuevas formas de socialización que promueven las TIC y sus implicancias para el desarrollo y la conformación de la identidad, propiciando procesos de reflexión y formación de criterios para actuar al respecto.

4

4.1.3 Incorpora en el diseño e implementación de las actividades con TIC's, principios de la ciudadanía digital para el acceso y uso de la información y para la configuración de prácticas sociales digitales. 


\begin{tabular}{|c|c|}
\hline Competencias & Criterios \\
\hline $\begin{array}{l}4.2 \text { Incorporar TIC con- } \\
\text { forme a prácticas que fa- } \\
\text { vorezcan el respeto a la di- } \\
\text { versidad, igualdad de trato, } \\
\text { y condiciones saludables en } \\
\text { el acceso y uso. }\end{array}$ & $\begin{array}{l}\text { 4.2.1 Integra, en el trabajo con TIC, estrategias que aseguren para todos } \\
\text { los estudiantes un acceso equitativo a los recursos tecnológicos y digi- } \\
\text { tales, procurándoles las mejores condiciones disponibles y atendiendo a } \\
\text { sus capitales culturales y diversas capacidades. } \\
\text { 4.2.2 Incluye procedimientos de prevención y cuidado de la salud de los } \\
\text { estudiantes y del ambiente educativo al trabajar con TIC. } \\
\text { 4.2.3 Evalúa los logros alcanzados en las acciones implementadas para } \\
\text { favorecer el acceso equitativo a los recursos tecnológicos y digitales, y } \\
\text { la prevención y cuidado de la salud de los/las estudiantes y del medio } \\
\text { ambiente. }\end{array}$ \\
\hline $\begin{array}{l}4.3 \text { Incorporar TIC confor- } \\
\text { me a prácticas que favorez- } \\
\text { can el cumplimiento de las } \\
\text { normas éticas y legales. }\end{array}$ & $\begin{array}{l}\text { 4.3.1 Modela y aplica, en las experiencias de aprendizaje en que se utili- } \\
\text { cen TIC, prácticas de reflexión y de toma de decisiones respecto a dile- } \\
\text { mas éticos y legales relacionados con su uso. } \\
\text { 4.3.2 Modela, planifica e implementa actividades que propicien conduc- } \\
\text { tas respetuosas de las normas éticas y legales en el uso de TIC, especial- } \\
\text { mente en la prevención y tratamiento del acoso (bullying) y de fenóme- } \\
\text { nos relacionados. }\end{array}$ \\
\hline
\end{tabular}

\section{DIMENSIÓN DESARROLLO Y RESPONSABILIDAD PROFESIONAL}

\section{Competencias \\ 5.1. Usar TIC en las ac-} tividades de formación continua y de desarrollo profesional, participando en comunidades de aprendizaje presencial o virtual y a través de otras estrategias no formales apropiadas para el desarrollo de este tipo de competencias.

\section{Criterios}

5.1.1 Selecciona y participa en actividades de formación continua sobre el uso e integración de las TIC en temas pedagógicos y de contenidos propios del sector de aprendizaje.

5.1.2 Participa en comunidades de aprendizaje presenciales o virtuales ligadas a su quehacer profesional, utilizándolas como una oportunidad de aprendizaje y desarrollo profesional.

5.1.3 Usa estrategias no formales para el desarrollo de competencias TIC, orientando este esfuerzo a la integración de las TIC en las prácticas pedagógicas y en los contenidos propios del sector de aprendizaje. 
5.2 Aplicar estrategias y procesos para la gestión de conocimiento mediado por TIC, con el fin de mejorar la práctica docente y el propio desarrollo profesional.
5.2.1 Integra las TIC de manera pertinente en el quehacer y desarrollo profesional, usándolas para la obtención, almacenamiento y organización de información.

5.2.2 Intercambia con sus pares reflexiones, experiencias y recursos sobre y para el uso de las TIC en su desarrollo profesional.

5.2.3 Usa TIC para la comunicación y colaboración con sus pares para fines de gestión curricular.

\begin{tabular}{|l|l|}
\hline Competencias & Criterios \\
\hline 5.3 Reflexionar sobre los \\
resultados del uso y ma- \\
$\begin{array}{l}\text { 5.3.1 Utiliza metodología de análisis para la reflexión de su prác- } \\
\text { tica con uso de TIC. }\end{array}$ \\
$\begin{array}{l}\text { 5.3.2 Participa en instancias de evaluación y autoevaluación sobre } \\
\text { el manejo instrumental de TIC para diagnosticar su nivel de do- } \\
\text { diseñando e implemen- } \\
\text { tando acciones de mejo- } \\
\text { ra. }\end{array}$ & $\begin{array}{l}\text { 5.3.3. Define un itinerario de desarrollo profesional asociado al } \\
\text { uso yanejo de TIC. } \\
\mathbf{5 . 3 . 4} \text { Diseña e implementa acciones de mejoramiento para el que- } \\
\text { hacer profesional a partir de la reflexión sobre el uso y manejo de } \\
\text { TIC. }\end{array}$ \\
\hline
\end{tabular}

\subsection{Habilidades TIC para el Aprendizaje (HTPA)}

Por otra parte se requiere que los docentes del sistema escolar se hagan cargo de la formación de las llamadas "Habilidades TIC para el Aprendizaje" (HTPA), de los escolares chilenos. ENLACES del Ministerio de Educación (2013), las define como: "La capacidad de resolver problemas de información, comu- nicación y conocimiento, así como dilemas legales, sociales y éticos en ambiente digital".

Las habilidades TIC han sido organizadas en dimensiones y sub-dimensiones, a saber:

a. Dimensión Información: en ella se describe las habilidades para buscar, seleccionar, evaluar y organizar información en entornos digitales y transformar o adaptar la informa-

Volumen No. 1 Integración de TIC en la Formación Inicial Docente de la Universidad de Los Lagos 
ción en un nuevo producto, conocimiento o desarrollar ideas nuevas. Se conforma por las sub-dimensiones:

- Información como fuente

- Información como producto

\section{b. Dimensión Comunicación y Colabora-} ción: definidas como "habilidades sociales, donde la capacidad para transmitir e intercambiar información e ideas con otros, así como también de interactuar y contribuir dentro de un grupo o comunidad es fundamental". Constituida por las sub-dimensiones:

- Comunicación Efectiva

- Colaboración

c. Dimensión Convivencia Digital: se refiere a las habilidades para la convivencia digital, como relacionarse a través de las redes sociales y resguardarse de situaciones riesgosas en Internet. Conformada por las subdimensiones:

- Ética y Autocuidado

- $\quad$ TIC y Sociedad

d. Dimensión Tecnología: son las habilidades funcionales y conocimientos necesarios para nombrar, resolver problemas, operar y usar las TIC en cualquier tarea. Compuesta por tres sub-dimensiones, a saber:

- Conocimientos TIC

- Operar las TIC

- Usar las TIC

En toda formación pedagógica en Chile debería considerarse preparar a los futuros docentes para planificar, ejecutar y evaluar actividades de aprendizaje que aborden el desarrollo de las mencionadas habilidades TIC.

\section{Metodología}

Se conformó una comisión constituida por académicos de la universidad que tuvieron a su cargo asignaturas o módulos de la línea TIC en Educación, representada por integrantes de ambos Campus. Se realizó un estudio de caso, cuya muestra fueron trece carreras de pedagogía impartidas por la Universidad de Los Lagos, entre los Campus de la ciudad de Osorno y la ciudad de Puerto Montt, al sur de Chile. De las trece carreras, diez de ellas correspondieron al Campus Osorno y tres al Campus Puerto Montt, como se puede apreciar a continuación: 
Tabla $N^{\circ} 1$. Distribución de carreras por campus

\begin{tabular}{|c|c|}
\hline Campus Puerto Montt & Campus Osorno \\
\hline $\begin{array}{l}\text { 1. Pedagogía Educación media en Artes, } \\
\text { menciones Artes Visuales - Música }\end{array}$ & $\begin{array}{l}\text { 1. Pedagogía Educación media en Matemáticas y } \\
\text { Computación }\end{array}$ \\
\hline $\begin{array}{l}\text { 2. Pedagogía Educación media en Educación } \\
\text { Física }\end{array}$ & $\begin{array}{l}\text { 2. Pedagogía Educación media en Lengua Castellana } \\
\text { y comunicación }\end{array}$ \\
\hline $\begin{array}{l}\text { 3. Pedagogía Educación Básica, mención } \\
\text { Inglés }\end{array}$ & $\begin{array}{l}\text { 3. Pedagogía Educación media en Inglés y } \\
\text { Traducción }\end{array}$ \\
\hline & $\begin{array}{l}\text { 4. Pedagogía Educación media en Historia y } \\
\text { Geografía }\end{array}$ \\
\hline & $\begin{array}{l}\text { 5. Pedagogía Educación media en Biología y } \\
\text { Química }\end{array}$ \\
\hline & 6. Pedagogía Educación media en Educación Física \\
\hline & $\begin{array}{l}\text { 7. Pedagogía Básica, mención Ciencias de la } \\
\text { Naturaleza }\end{array}$ \\
\hline & 8. Pedagogía Básica, mención Matemáticas \\
\hline & $\begin{array}{l}\text { 9. Educación Diferencial con especialización en } \\
\text { problemas de aprendizaje }\end{array}$ \\
\hline & 10. Educación Parvularia \\
\hline
\end{tabular}

Para la recolección de información se utilizó la técnica de documentación. Con ella se analizaron las mallas curriculares de cada carrera y sus respectivos programas de asignaturas TIC (en Educación). Sólo quedaron exentos de este último análisis, los programas de asignaturas a los cuales fue imposible acceder en las carreras del Campus Osorno, tales como: Pedagogía en Educación Física y Educación Parvularia.

Para el análisis de las mallas se elaboró una matriz para identificar las asignaturas, sus créditos y horas de trabajo directo del estudiante en cada una de las carreras. Con esa información se obtuvo una primera aproximación a la situación, a partir de la cual se comienzan a solicitar los programas de estudio de cada una de las asignaturas o módulos que se consideraba que tributaban directamente con la formación de TIC en Educación. Los programas fueron solicitados a jefes de carrera y docentes a cargo de su implementación. Una vez obtenida esa información se elaboró una nueva matriz que corroborara la existencia de los criterios que componen las

Volumen No. 1 Integración de TIC en la Formación Inicial Docente de la Universidad de Los Lagos 
cinco dimensiones de las competencias TIC definidas en Chile para la formación inicial docente. Para el llenado de la matriz se asignaron colores para identificar el nivel de presencia de contenido relativo a un criterio. Es así como el color verde significaba presencia; el color amarillo relativa presencia y el color rojo ausencia de ese criterio. Una vez identificada visualmente la situación, se otorgaron valores numéricos asociados a la presencia con la finalidad de graficar:

- PRESENCIA: presencia mayoritaria del criterio en los programas de estudio de la carrera. (valor 1 punto)

- PARCIALMENTE: el criterio se presentó de forma minoritaria en los programas de estudio de la carrera. (valor 0 punto)

- AUSENCIA: total y absoluta ausencia del criterio en los programas de estudio de la carrera. (valor 0 punto)
- SIN INFORMACIÓN: no se tuvo acceso a información de los programas de estudio, por lo que no se pudo observar el comportamiento del criterio TIC en FID. (valor 0 punto)

\section{Resultados}

El análisis de mallas curriculares permitió observar la diversidad de contenidos, números de cursos existentes, número de horas asignadas en forma heterogénea, inorgánica de contenidos en carreras pre escolar, Educación General Básica y Educación Media. Los resultados arrojados de estos análisis son los siguientes:

\subsection{Análisis Cuantitativo}

En primera instancia se presentan los hallazgos en formato de tablas y gráficos, para cuantificar la caracterización del estado de las TIC en FID en la Universidad.

Tabla N $\mathbf{N}^{\circ}$. Cantidad de horas por asignaturas en carreras de Enseñanza Media en el Campus Osorno

\begin{tabular}{|l|c|c|}
\hline \multicolumn{1}{|c|}{ CARRERA } & $\begin{array}{c}\text { CANTIDAD } \\
\text { ASIGNATURAS }\end{array}$ & CANTIDAD \\
\hline Matemáticas y Computación & 6 & 13 \\
\hline Lengua Castellana y comunicación & 4 & 8 \\
\hline Inglés y Traducción & 4 & 8 \\
\hline Historia y Geografía & 4 & 8 \\
\hline Biología y Química & 4 & 6 \\
\hline Educación Física & 2 & 2 \\
\hline
\end{tabular}


[ 24 ] Universidad Católica de El Salvador.

Gráfico $\mathrm{N}^{\circ} 1$. Cantidad de horas y asignaturas TIC en Pedagogías en Educación media

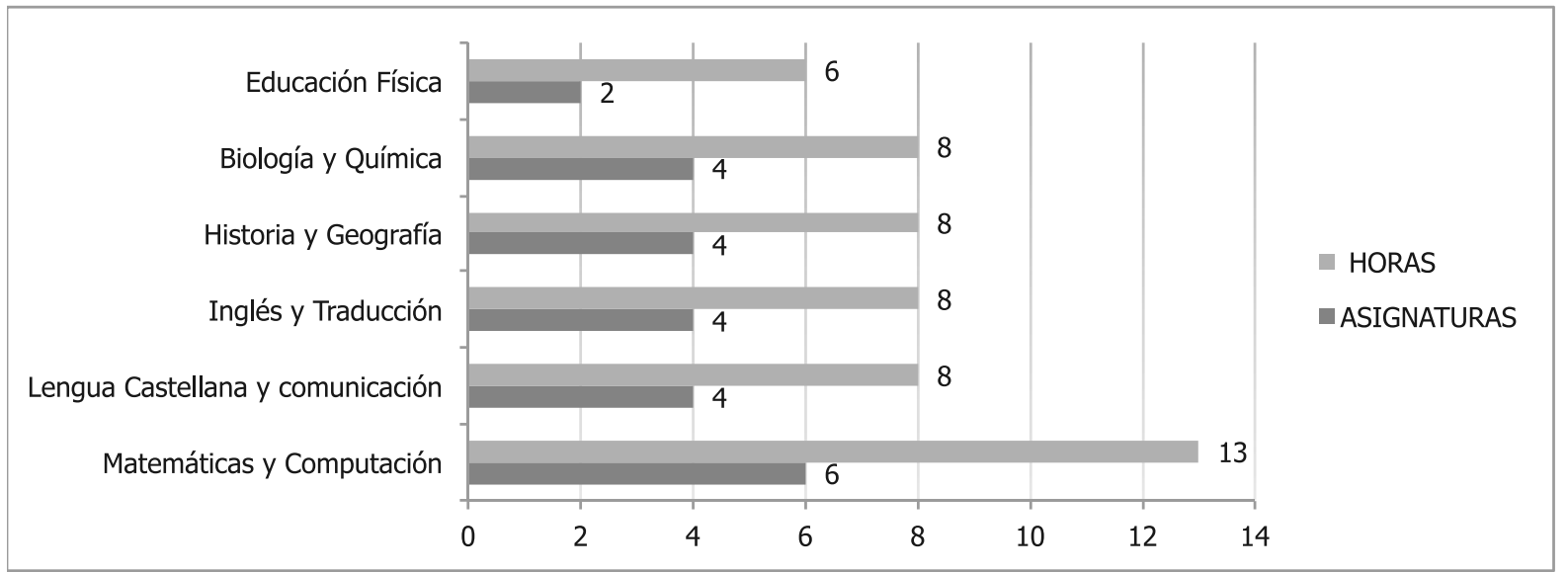

Tabla $N^{\circ} 3$. Cantidad de horas por asignaturas en carreras de Enseñanza Básica en el Campus Osorno

\begin{tabular}{|l|c|c|}
\hline \multicolumn{1}{|c|}{ CARRERA } & $\begin{array}{c}\text { CANTIDAD } \\
\text { ASIGNATURAS }\end{array}$ & HORAS \\
\hline Básica, mención Ciencias de la Naturaleza & 2 & 6 \\
\hline Básica, mención Matemáticas & 2 & 4 \\
\hline Diferencial con especialización en problemas de aprendizaje & 5 & 10 \\
\hline Educación Parvularia & 3 & 8 \\
\hline
\end{tabular}

Gráfico $\mathrm{N}^{\circ}$ 2. Cantidad de horas y asignaturas de TIC en las carreras de Educación Parvularia, Pedagogía en Educación Básica, y Educación Diferencial

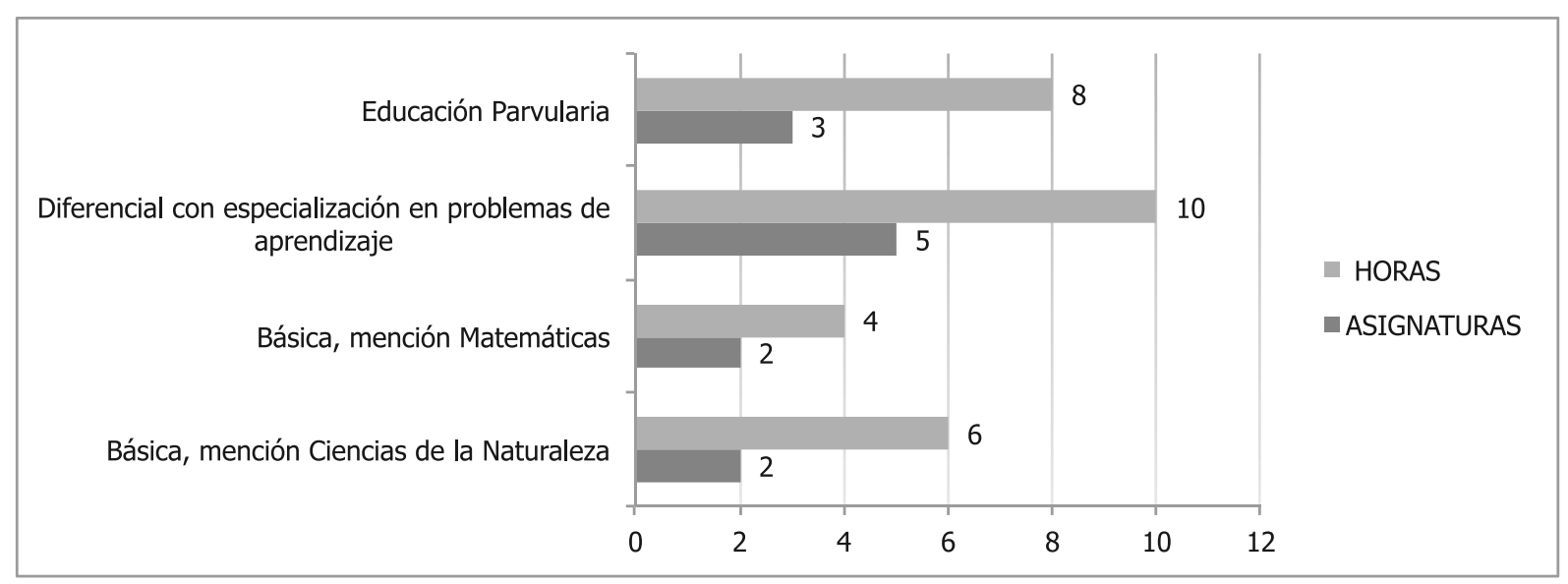

Volumen No. $1 \mid$ Integración de TIC en la Formación Inicial Docente de la Universidad de Los Lagos 
Tabla N4. Cantidad de horas por asignaturas en carreras de Enseñanza Media y Básica en el Campus Puerto Montt

\begin{tabular}{|l|c|c|}
\hline \multicolumn{1}{|c|}{ CARRERA } & $\begin{array}{c}\text { CANTIDAD } \\
\text { ASIGNATURAS }\end{array}$ & $\begin{array}{c}\text { CANTIDAD HO- } \\
\text { RAS }\end{array}$ \\
\hline Artes, menciones Artes Visuales - Música & 1 & 2 \\
\hline Educación Física & 2 & 6 \\
\hline Básica. Mención Inglés & 1 & 2 \\
\hline
\end{tabular}

Gráfico $\mathrm{N}^{\circ}$ 3. Cantidad de horas y asignaturas Carreras de Pedagogía en el Campus Puerto Montt

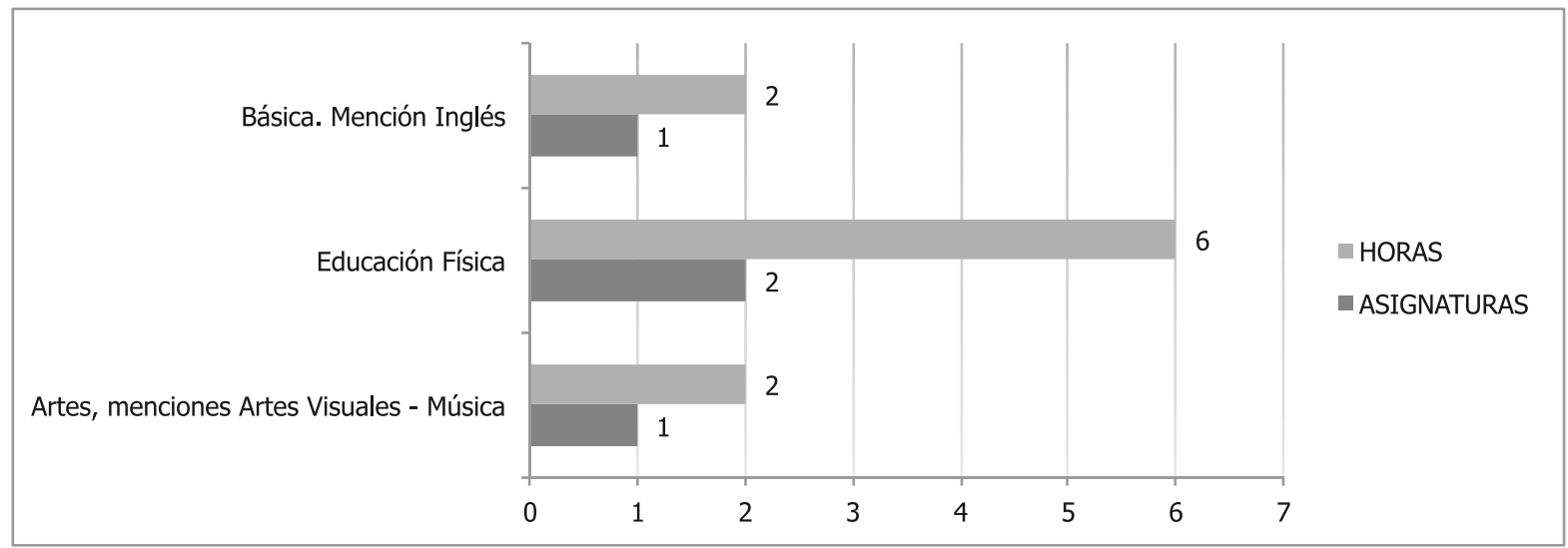

\subsection{Análisis Cualitativo}

\subsubsection{Análisis de mallas Curriculares}

De acuerdo al análisis relativo a la cantidad de asignaturas y horas que tributan a las TIC en educación, en los dos Campus, se desprende lo siguiente:

- Amplia heterogeneidad en los programas de estudio, tanto en los contenidos abordados como en el nivel de profundidad de los mismos.

- Tendencia a la reducción en el núme- ro de asignaturas y horas TIC según los nuevos decretos de carreras. Esto permite suponer que la necesidad obedece a ajustar el número de créditos a la totalidad de la malla y no a un criterio pedagógico respaldado por un especialista en el área de TIC; traduciéndose en una debilidad para las carreras pedagógicas.

- Se presenta un número importante de asignaturas cuya coincidencia entre los nombres decretados y los que aparecen 
en el programa de estudio no existe, generando trastornos en los procesos administrativos internos.

- Disparidad en los contenidos dada la diferencia en el número de asignaturas. Queda en evidencia la distinta formación en TIC que reciben estudiantes de pedagogía de un mismo Campus y entre Campus.

- No todas las carreras pedagógicas privilegian la formación en TIC para favorecer los procesos de enseñanza-aprendizaje, también se encuentran aquellas que privilegian la formación en ofimática.

- Se presenta un sistema diverso de contenidos: de código abierto y pago. Es necesario establecer software y aplicaciones comunes para las carreras pedagógicas, en especial aquellas que pueden estar al acceso de los futuros docentes en el sistema educativo.
- Se evidencia la aplicación práctica de las TIC en ambientes educativos en seis carreras del Campus Osorno y en una dentro del Campus Puerto Montt.

- No existe coordinación entre los docentes que dictan las asignaturas de TIC en los distintos Campus. Esto se resolvió gracias a la creación del grupo de investigación Huilli-TIC.

\subsubsection{Análisis de Programas de Estudio en relación a Competencias TIC}

Los siguientes resultados muestran presencia de los criterios de las competencias TIC en FID. El grupo de docentes especialistas pueden corroborarlo de acuerdo a su experiencia en la ejecución de los programas de estudio y sus contenidos, aunados a su desarrollo en el aula.

La tabla refleja los cuarenta y tres criterios buscados en cada programa de estudio. Asimismo se muestran las carreras a cuya información no se tuvo acceso. 
Tabla $N^{\circ} 5$. Presencia de Competencias TIC en carreras de Enseñanza Media en el campus Osorno

\begin{tabular}{|l|c|c|c|c|}
\hline \multirow{2}{*}{ CARRERAS CAMPUS OSORNO } & \multicolumn{4}{|c|}{ CANTIDAD DE CRITERIOS/DIMENSIONES PRESENTES } \\
\cline { 2 - 5 } Matemáticas y Computación & PRESENCIA & PARCIALMENTE & AUSENCIA & SIN INFORMACION \\
\hline Lengua Castellana y comunicación & 26 & 1 & 0 & $42^{*}$ \\
\hline Inglés y Traducción & 26 & 11 & 6 & 0 \\
\hline Historia y Geografía & 26 & 11 & 6 & 0 \\
\hline Biología y Química & 26 & 11 & 6 & 0 \\
\hline Educación Física & 0 & 0 & 0 & 0 \\
\hline
\end{tabular}

Tabla $N^{\circ} 6$. Presencia de Competencias TIC en carreras de Enseñanza Básica en el campus Osorno

\begin{tabular}{|l|c|c|c|c|}
\hline \multirow{2}{*}{ CARRERAS CAMPUS OSORNO } & \multicolumn{4}{|c|}{ CANTIDAD DE CRITERIOS/DIMENSIONES PRESENTES } \\
\cline { 2 - 5 } & PRESENCIA & PARCIALMENTE & AUSENCIA & $\begin{array}{c}\text { SIN } \\
\text { INFORMACION }\end{array}$ \\
\hline $\begin{array}{l}\text { Básica, mención Ciencias de la } \\
\text { Naturaleza }\end{array}$ & 12 & 21 & 6 & $4^{*}$ \\
\hline Básica, mención Matemáticas & 12 & 21 & 6 & $4^{*}$ \\
\hline $\begin{array}{l}\text { Diferencial con especialización en } \\
\text { problemas de aprendizaje }\end{array}$ & 26 & 11 & 6 & $6^{*}$ \\
\hline Educación Parvularia & 9 & 8 & 3 & $23^{*}$ \\
\hline
\end{tabular}

Tabla $N^{\circ}$ 7. Presencia de Competencias TIC en carreras de Enseñanza Básica y Media en el campus Puerto Montt

\begin{tabular}{|l|c|c|c|c|}
\hline \multirow{2}{*}{$\begin{array}{l}\text { CARRERAS CAMPUS } \\
\text { PUERTO MONTT }\end{array}$} & \multicolumn{3}{|c|}{ CANTIDAD DE CRITERIOS/DIMENSIONES PRESENTES } \\
\cline { 2 - 5 } & PRESENCIA & PARCIALMENTE & AUSENCIA & $\begin{array}{c}\text { SIN INFORMA- } \\
\text { CION }\end{array}$ \\
\hline $\begin{array}{l}\text { Artes, menciones Artes Visua- } \\
\text { les - Música }\end{array}$ & 4 & 21 & 18 & 0 \\
\hline Educación Física & 30 & 11 & 2 & 0 \\
\hline Básica. Mención Inglés & 4 & 21 & 18 & 0 \\
\hline
\end{tabular}


A continuación se desglosa el análisis según la situación de cada dimensión de las competencias TIC.

\section{a. Dimensión Pedagógica}

La dimensión Pedagógica plantea que los futuros docentes deben adquirir y demostrar formas de aplicar las TIC en el currículum escolar vigente como una forma de apoyar y expandir el aprendizaje y la enseñanza.

\section{Gráfico $\mathrm{N}^{\circ} 4$. Situación Dimensión Pedagógica}

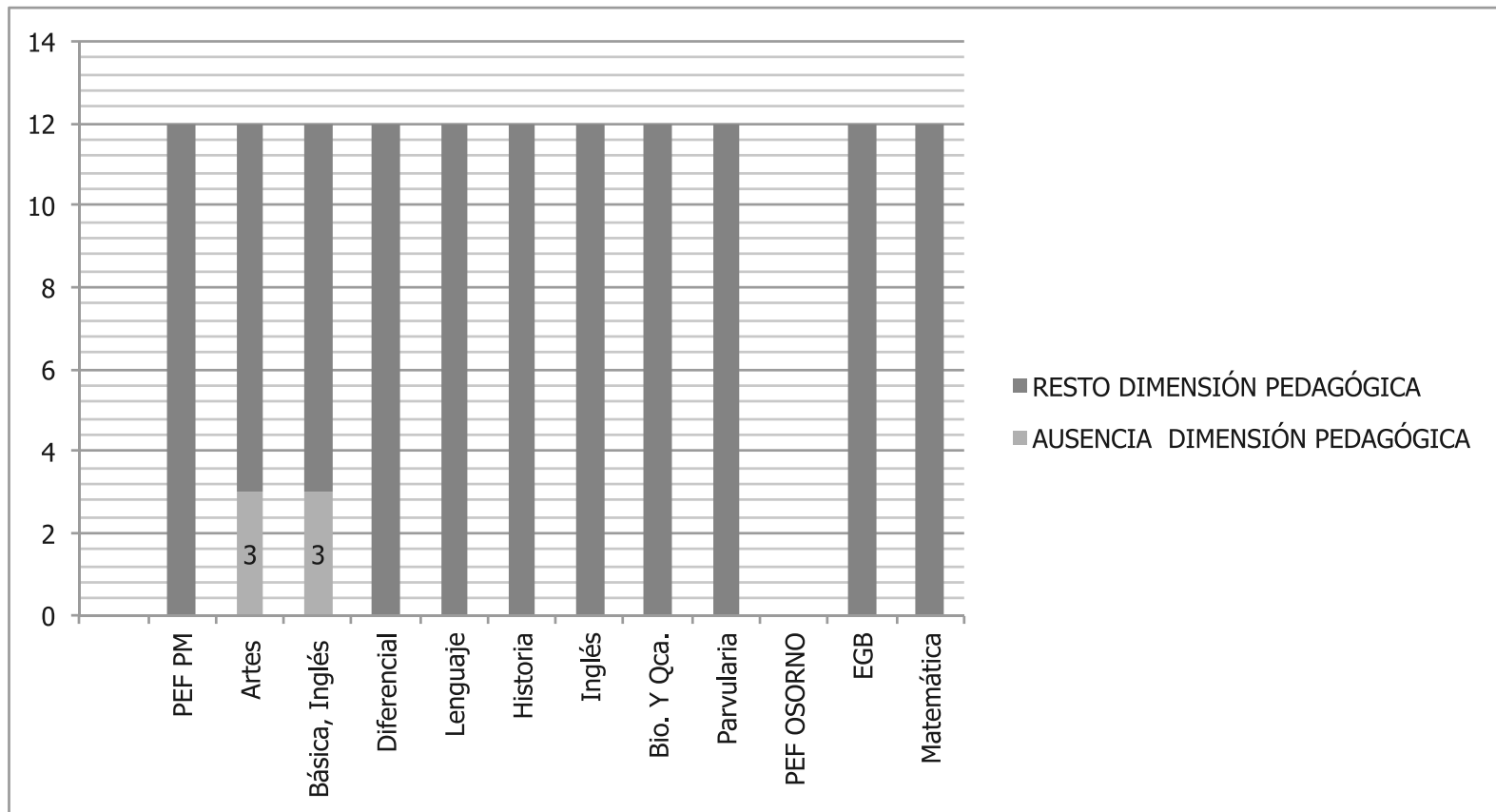

A través del siguiente gráfico se puede observar que en dos de las carreras del campus Puerto Montt existe ausencia de 3 de los 12 criterios. Ello que se debe al reducido tiempo que se destina a esta área en ambas carreras. En el caso de la carrera de Artes solo hay una asignatura de Informática Educativa de dos horas en toda la formación; mientras que en la carrera de Básica, mención Inglés solo existen dos módulos de dos horas cada uno.

\section{b. Dimensión Técnica}

En esta dimensión se evalúa si los futuros docentes demuestran un dominio de las competencias asociadas al conocimiento general de las TIC y el manejo de las herramientas de productividad (procesador de texto, hoja de cálculo, presentador) e Internet, desarrollando habilidades y destrezas para el aprendizaje permanente de nuevos hardware y software. 


\section{Gráfico $\mathrm{N}^{\circ}$ 5. Situación Dimensión Técnica}

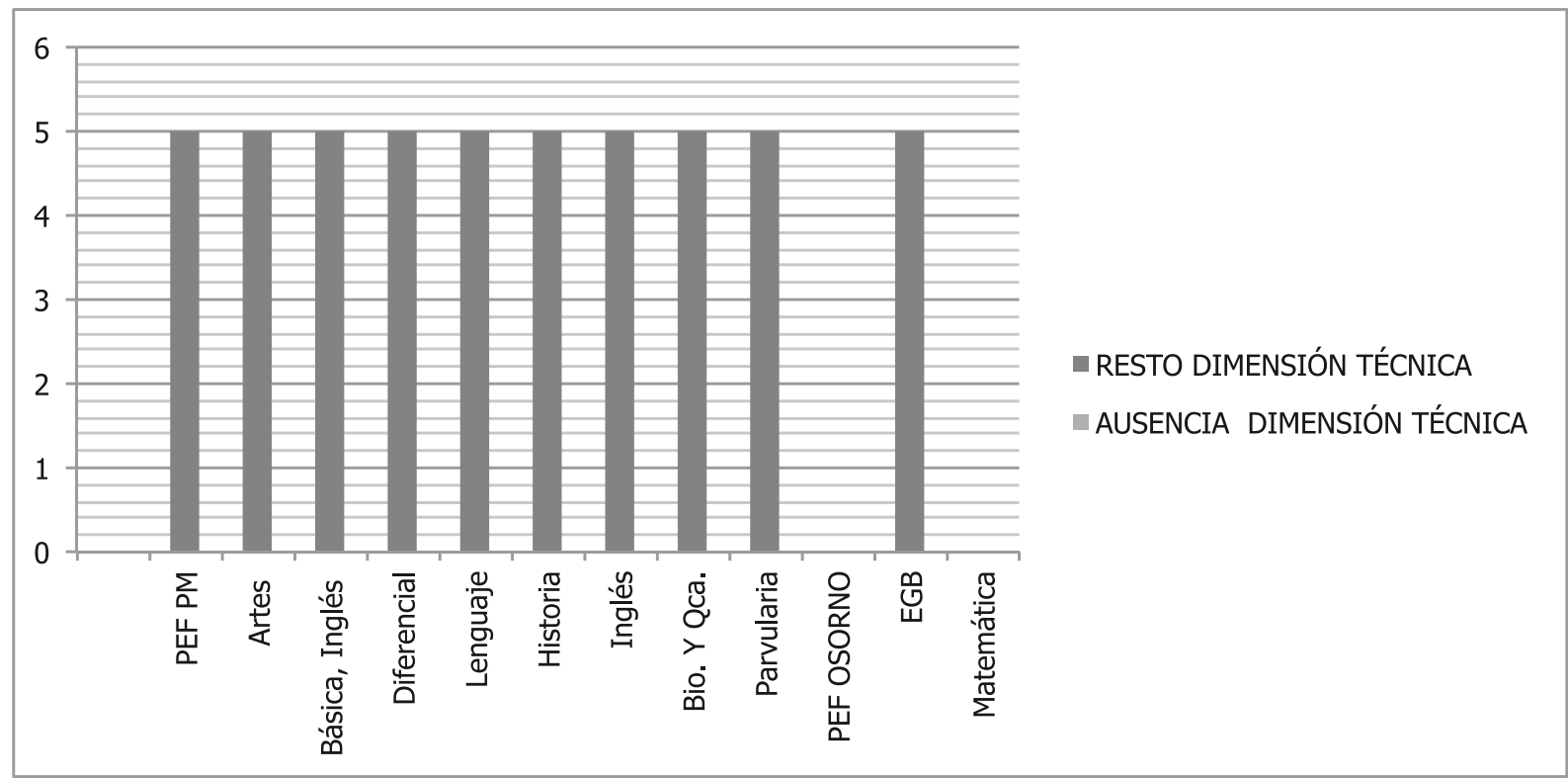

En general todas las carreras (según los programas de estudio) manifiestan que desarrollan todos los criterios que fueron tomados en esta dimensión.

\section{c. Dimensión Gestión}

El área de gestión hace referencia a que los futuros docentes hagan uso de las TIC para apoyar su trabajo en el área administrativa, tanto a nivel de su gestión docente como de apoyo a la gestión del establecimiento.

\section{Gráfico $\mathrm{N}^{\circ} 6$. Situación Dimensión Gestión}

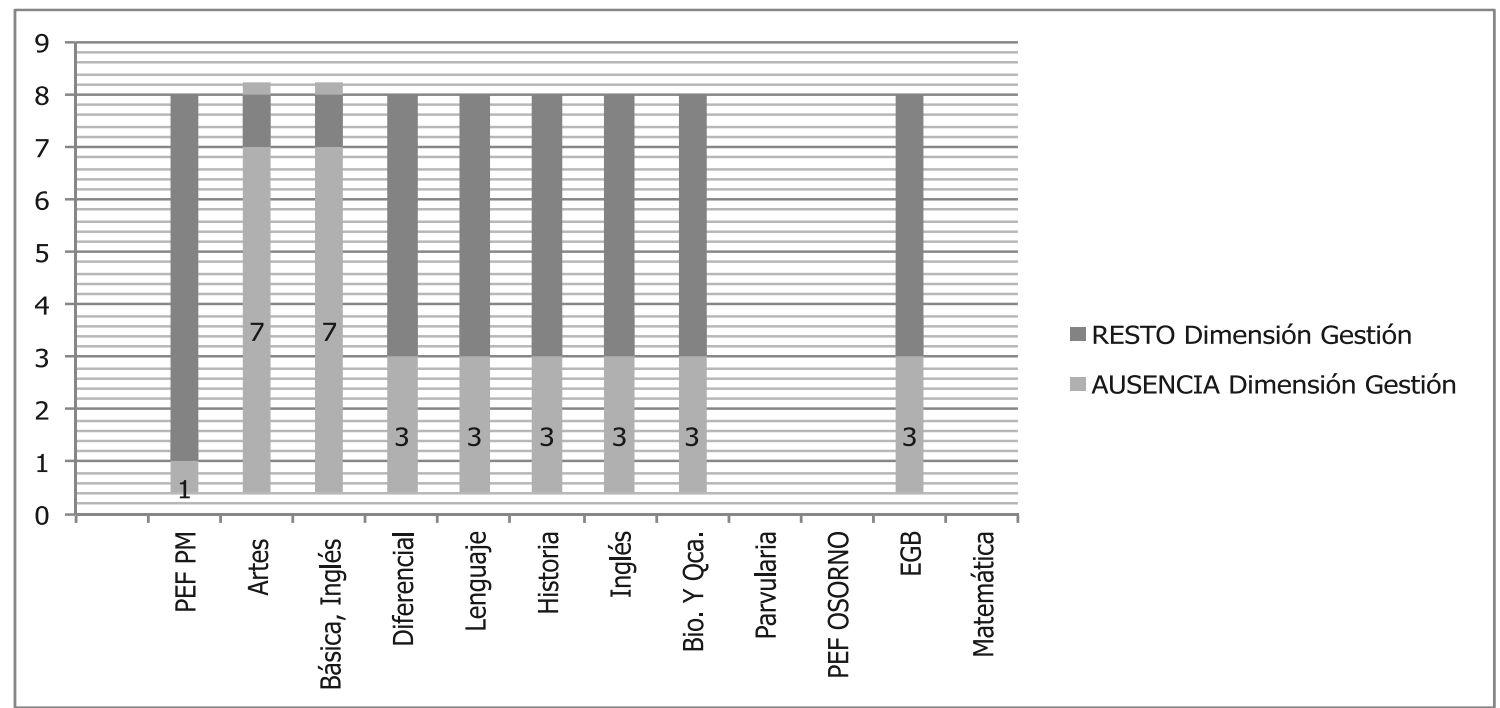

Brenda Angélica Lara Subiabre / Yorka Tatiana Ortiz Ruiz / Pedro Marcelo Soto Alarcón / Francisco Javier Núñez Romero | Volumen No. 1 Marisa Alejandra Lara Escobar / Francisco José Kroff Trujillo 
Como se puede apreciar en el gráfico, esta dimensión fue la que presentó mayores deficiencias. Nuevamente dos de las carreras de Puerto Montt tienen una alta ausencia de criterios trabajados en la formación, debido a la falta de asignaturas y créditos destinados para ello. No así en el caso de Educación Física, que trabaja esa área desde las prácticas profesionales.

\section{d. Dimensión Ética, Social y Legal}

En esta dimensión se debe evidenciar que los futuros docentes conocen, se apropian y difunden entre sus estudiantes los aspectos éticos, legales y sociales relacionados con el uso de los recursos informáticos y contenidos disponibles en Internet, actuando de manera consciente y responsable respecto de los derechos, cuidados y respetos que deben considerarse en el uso de las TIC.

\section{Gráfico $\mathrm{N}^{\circ} 7$. Situación Dimensión Ética, Social y Legal}

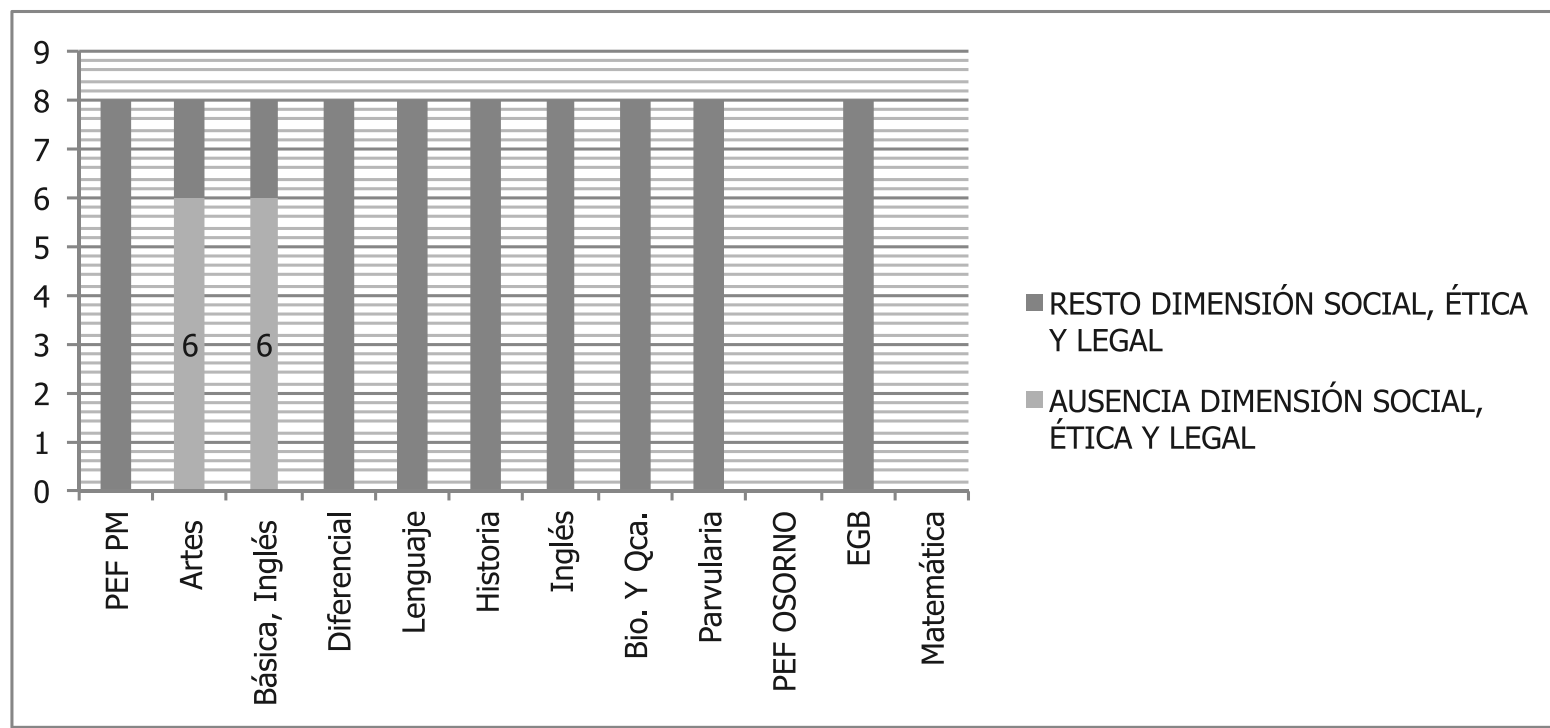

El gráfico revela que dos de las carreras de Puerto Montt presentan alta deficiencia de criterios en el análisis realizado.

\section{e. Dimensión Desarrollo y Responsabili- dad Profesional}

Los futuros docentes hacen uso de las TIC como medio de especialización y desarrollo profesional, informándose y accediendo a diversas fuentes para mejorar sus prácticas y facilitando el intercambio de experiencias que contribuyan mediante un proceso de reflexión con diversos actores educativos, a conseguir mejores procesos de enseñanza y aprendizaje. 


\section{Gráfico N ${ }^{\circ}$. Situación Dimensión Desarrollo y Responsabilidad Profesional}

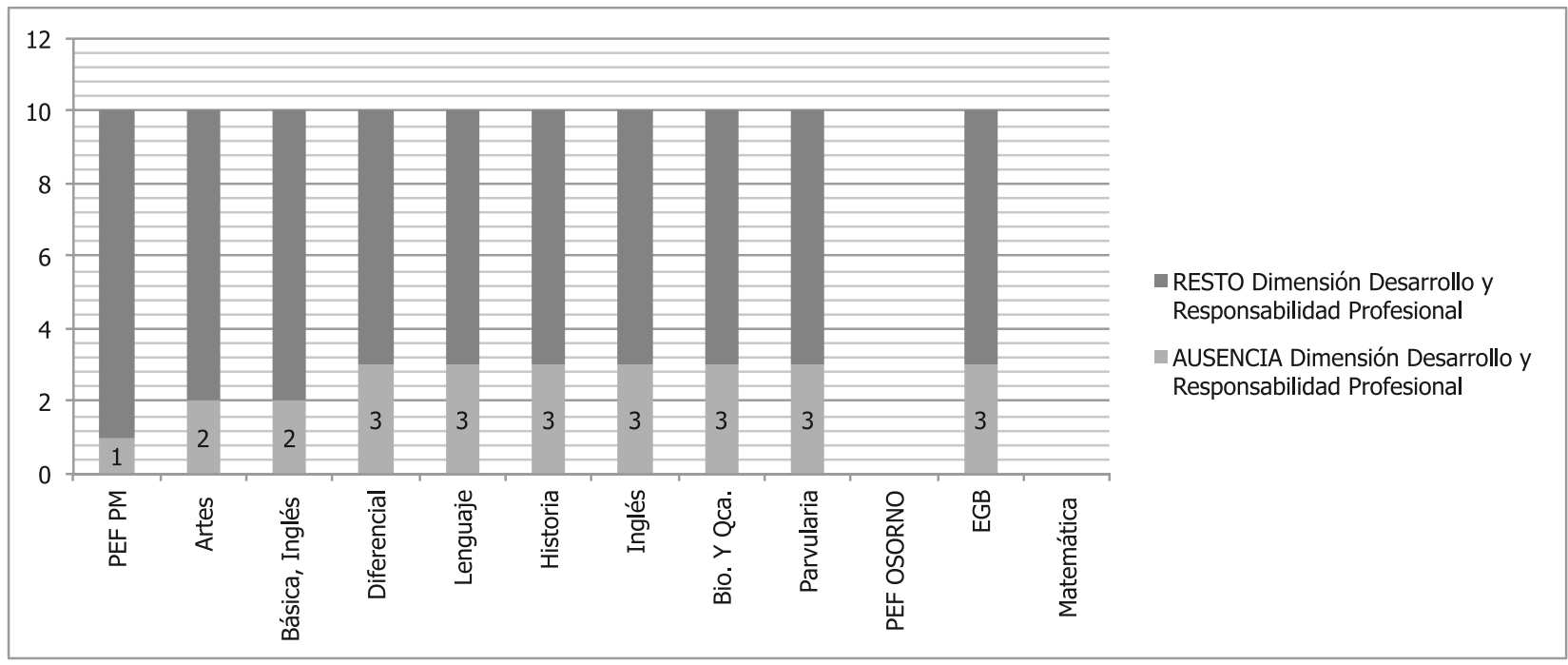

Se observa que el grado de integración en la formación es bastante similar en todas las carreras. En el caso de Puerto Montt se desarrolla un poco más, debido a que en los últimos años se ha comenzado a incorporar el trabajo con portafolio electrónico.

\section{Discusión}

El estudio permitió develar las condiciones en las que se están formando los estudiantes de pedagogía en relación al área de TIC en educación, principalmente a nivel curricular, que generalmente se caracteriza por ser heterogénea. Haber realizado una revisión exhaustiva de las mallas curriculares y los programas de estudio posibilitó que el equipo de investigación pudiera conocer cuál es el escenario del que se dispone para desarrollar las competencias TIC demandadas a los docen- tes, para luego generar las transformaciones que se requieran.

Si bien hemos avanzado, hacer la relación de los programas de estudio con los criterios de las competencias TIC, demuestra que la dimensión de gestión está débilmente abordada.

Considerando el contexto de trabajo desarrollado a partir de los intereses de conocer para armonizar líneas de acción al interior de las pedagogías, el desarrollo académico de la Universidad de los Lagos y el análisis de lo expuesto en esta investigación se plantean diferentes acciones de tipo formativas, de armonización a nivel curricular, de integración coordinada y asesoramiento para el uso de las nuevas tecnologías de la información y comunicación al interior de las carreras peda- 
gógicas. Este planteamiento orientará hacia propuestas de políticas educativas que permitan una implementación exitosa y eficaz de las TIC por parte de la Universidad de Los Lagos. De esta forma se responderá a las exigencias externas, como la Prueba Inicia TIC, que pone a los estudiantes y a las instituciones en una situación compleja de afrontar, pero que sin duda se debe enfrentar.

Es así como el equipo de trabajo estableció condiciones indispensables o acuerdos para desarrollar en forma paulatina, pero eficaz el respectivo respaldo institucional, que avala su integración como una línea sólida para la formación de los futuros pedagogos egresados de la Universidad de Los Lagos. Esta propuesta tiene que ver con:

- Participar de los procesos de armonización curricular de las carreras pedagógicas como una forma de garantizar el contar con los tiempos y espacios adecuados para responder a las exigencias en la formación de un docente egresado de la U lagos.

- Generar instrumentos que permitan determinar las competencias TIC de egreso e ingreso de los estudiantes de las carreras pedagógicas.

- Establecer un proyecto piloto para determinar las posibilidades reales de vincular la línea de didáctica de las carreras pedagógicas a la línea de informática educativa.
- Vincular la integración de TIC los procesos de prácticas profesionales como espacios para confirmar su incorporación en contextos reales y poder orientar el trabajo cuando así sea requerido.

- Consolidar equipos de trabajo, conformado por profesionales de los Campus Osorno y Puerto Montt, que permitan enfrentar en forma coordinada el proceso de consolidación de la línea de informática educativa.

Entre las principales limitaciones para el desarrollo del estudio, se encontraron dificultades para reunir al equipo de trabajo y el acceso a programas de estudio. En el caso de las sesiones de trabajo, el problema se debió a la gran carga docente que tiene cada uno de los integrantes y por el hecho de estar ubicados en ciudades diferentes, separados por más de cien kilómetros. Por otra parte, el acceso a programas de estudio obedece a que no hay instancias formales que los resguarden almacenar y actualicen anualmente; teniéndolos disponibles para el conocimiento público. Ello obliga a depender de las voluntades de académicos para facilitar los programas que implementan.

A partir del trabajo realizado, surgen algunas interrogantes ¿Qué competencias TIC han desarrollado los estudiantes al finalizar su formación pedagógica? y ¿Cuál es el estándar que debería definir la Universidad para garantizar el desarrollo de las competencias TIC en sus estudiantes? 


\section{Referencias}

Brun, M. (2011). Las Tecnologías de la Información y la Comunicación en la Formación Inicial Docente en América Latina". CEPAL, División de Desarrollo Social. Serie políticas sociales $\mathrm{N}^{\circ}$ 172. En: http://www.cepal.org/publicaciones/xml/2/44612/Serie_172_Mario_Brun_Tic_ ALIS_09.2011.pdf

Centro de Investigaciones y Desarrollo en Educación (CIDE). (2010). Diseño e Implementación de Estrategia Nacional para la Incorporación de TIC en la Formación Inicial Docente. Chile. En: http://www.cide.cl/asesoria/asesoria_desarrollo_detalle_diseno_implementacion_tic.php

ENLACES, Ministerio de Educación. (2008). Estándares TIC para la Formación Inicial Docente, una Propuesta en el Contexto Chileno. Chile. Ministerio de Educación.

ENLACES. Ministerio de Educación. (2013). Matriz de habilidades TIC para el Aprendizaje. En: http://www.enlaces.cl/index.php?t=44\&i=2\&cc $=2285 \& \mathrm{tm}=2$

Hepp, P. (2012). "Barreras para la Integración de las TIC en la Formación Inicial Docente". En: http://www.enlaces.cl/tp_enlaces/portales/tpe76eb4809f44/uploadImg/File/2012/ seminarios/fidtic/Seminario\%20FIDTIC\%20(Hepp).pdf

Mourshed, M. y Barber, M. (2010) Informe Mckinsey “how the world's best-performing school systems come out on top". Fuente: http://www.mckinsey.com/clientservice/social_sector/our_ practices/education/knowledge_highlights/how\%20school\%20systems\%20get\%20better.aspx

Programa IINICIA, Prueba de Habilidades TIC en Ambientes Pedagógicos, en http://www. programainicia.cl/ed04_tecnologias.html

Relpe. (2013). Caracterización de Buenas Prácticas en Formación Inicial Docente en TIC. En: http://www.relpe.org/wp-content/uploads/2013/04/12-Caracterizaci\%C3\%B3n-de-buenaspr\%C3\%A1cticas-en-formaci\%C3\%B3n-inicial-docente-en-TIC.pdf

Silva, J. y Astudillo, A. (2012). Inserción de TIC en la Formación Inicial Docente: Barreras y Oportunidades. Revista Iberoamericana de Educación, Volumen 4, N58. En: http://www. rieoei.org/rie_contenedor.php?numero=4557\&titulo=Inserci\%F3n\%20de\%20TIC\%20en\%20 la\%20formaci\%F3n\%20inicial\%20docente:\%20barreras\%20y\%20oportunidades

Brenda Angélica Lara Subiabre / Yorka Tatiana Ortiz Ruiz / Pedro Marcelo Soto Alarcón / Francisco Javier Núñez Romero | Volumen No. 1 Marisa Alejandra Lara Escobar / Francisco José Kroff Trujillo 